\title{
Fitossociologia de um trecho da mata ciliar do rio da Prata, Jardim, MS, Brasil ${ }^{1}$
}

\author{
Joanice Lube Battilani²,3, Edna Scremin-Dias² e Andréa Lúcia Teixeira de Souza ${ }^{2}$
}

Recebido em 28/09/2004. Aceito em 04/02/2005

\begin{abstract}
RESUMO - (Fitossociologia de um trecho da mata ciliar do rio da Prata, Jardim, MS, Brasil). O presente estudo teve como objetivos descrever a riqueza e a diversidade em espécies e analisar os aspectos fitossociológicos de um trecho da mata ciliar do rio da Prata, localizado na Fazenda Nossa Senhora Aparecida no município de Jardim, MS. Seis transectos de $150 \mathrm{~m}$ cada foram distribuídos aleatoriamente perpendiculares à margem do rio e subdivididos em 90 parcelas de $10 \mathrm{~m} \times 10 \mathrm{~m}$. Em cada parcela foram amostrados todos os indivíduos com diâmetro à $1,30 \mathrm{~m}$ de altura do solo (DAP) $\geq 3,18 \mathrm{~cm}$, totalizando 661 indivíduos pertencentes a 33 famílias, 57 gêneros e 66 espécies. As famílias Meliaceae, Mimosaceae, Sapindaceae, Euphorbiaceae, Fabaceae, Myrtaceae e Rutaceae destacaram-se pelo elevado número de espécies. Myrcianthes pungens (O. Berg) D. Legrand, Adelia membranifolia (Müll. Arg.) Chodat \& Hassl., Dalbergia sp., Holocalyx balansae Micheli, Unonopsis lindmanii R.E. Fr., Guarea kunthiana A. Juss., Trichilia claussenii C. DC., Guarea guidonea (L.) Sleumer, Averrhoidium paraguaiense Radlk.e Astronium graveolens Jacq. foram as espécies de maior valor de importância. O índice de diversidade de Shannon (H') obtido foi 3,413 nats/indivíduo e a eqüabilidade (J') foi 0,815. A densidade e a área basal estimada foi 734,44 indivíduos/ha e $21,32 \mathrm{~m}^{2} /$ ha respectivamente. Os resultados obtidos neste trabalho reforçam a necessidade de preservação da mata ciliar do rio da Prata e são de extrema importância para subsidiar projetos de manejo e recomposição nestas formações.
\end{abstract}

Palavras-chave: mata ciliar, comunidade arbóreo-arbustivo, fitossociologia, Serra da Bodoquena

\begin{abstract}
Phythossociologic along a riparian forest at rio da Prata, Jardim, Mato Grosso do Sul State, Brazil). The present study describes species richness and diversity, and analyses phythossociologic aspects of a riparian forest at Rio da Prata, Jardim district, Mato Grosso do Sul State, Brazil. The phytosociological study was carried out on six $150 \mathrm{~m}$ transects perpendicular to the river subdivided in 90 quadrats $(10 \mathrm{~m} \times 10 \mathrm{~m})$. All trees and shrubs with a minimum of $3,18 \mathrm{~cm}$ diameter at breast height were recorded, totalizing 661 individuals of 33 families, 57 genera and 66 species. The families with high number of species are Meliaceae, Mimosaceae, Sapindaceae, Euphorbiaceae, Fabaceae, Myrtaceae and Rutaceae. The species with the highest importance values are Myrcianthes pungens (O. Berg) D. Legrand, Adelia membranifolia (Müll. Arg.) Chodat \& Hassl., Dalbergia sp., Holocalyx balansae Micheli, Unonopsis lindmanii R.E. Fr., Guarea kunthiana A. Juss., Trichilia claussenii C. DC., Guarea guidonea (L.) Sleumer, Averrhoidium paraguaiense Radlk. and Astronium graveolens Jacq. The Shannon diversity index is H'=3,413 nats/individual and eqüability is J=0,815. The density and basal area are respectively 734,44 ind./ha and $21,32 \mathrm{~m}^{2} / \mathrm{ha}$. The results obtained in this study reinforce the protection of belt of riparian vegetation in the Rio da Prata, and will support management and restoration projects in these ecosystems.
\end{abstract}

Key words : riparian vegetation, community of trees and shrubs, phytossociologic, Serra da Bodoquena

\section{Introdução}

Dentre os diversos tipos fitofisionômicos do bioma Cerrado, as matas ciliares destacam-se por ocuparem áreas restritas ao longo dos cursos d'água de médio e grande porte, diferenciando-se das matas de galeria que acompanham pequenos rios, nascentes e córregos (Ribeiro \& Walter 1998; 2001). Estas formações são fortemente influenciadas por uma série de fatores físicos locais, como as variações edáficas e topográficas, além dos processos de perturbações naturais e antrópicos que são muito freqüentes nessas comunidades (Oliveira-Filho et al. 1994; Correia et al. 2001; Nebel et al. 2001; Campos \& Souza 2002). As matas ciliares, em geral, ocorrem em terrenos acidentados, não havendo uma transição evidente para outras fisionomias florestais como as matas decíduas e semidecíduas (Barbosa 2000). Esta associação com as formações adjacentes resulta numa composição florística mista, com espécies típicas das margens de rios e de outras formações adjacentes (Ivanauskas et al. 1997). No entanto, as matas ciliares diferenciam-se das formações adjacentes pela estrutura, em geral, mais densa e mais alta devido principalmente à

\footnotetext{
Parte da Dissertação de Mestrado da primeira Autora

Departamento de Biologia, Universidade Federal de Mato Grosso do Sul, C. Postal 549, CEP 79070-900, Campo Grande, MS, Brasil

Autora para correspondência: Rua Benício Pires de Freitas 354, Res. Itamarati, CEP 79240-000, Jardim, MS, Brasil (joanice@econet.com.br)
} 
associação com o curso d'água (Ribeiro \& Walter 1998). Levantamentos florísticos e fitossociológicos em remanescentes de florestas ciliares, realizados em diferentes regiões do Brasil, têm mostrado que essas áreas são muito diversas quanto à composição e estrutura fitossociológica como resultado da elevada heterogeneidade ambiental à qual estão associadas (Sanchez et al. 1999; Sampaio et al. 2000; Silva Júnior 2001a; Felfili et al. 2001).

As formações ciliares desempenham relevante importância na manutenção da integridade dos ecossistemas locais, representando importante áreas de preservação de espécies animais e vegetais e conservação dos recursos naturais (Montag et al. 1997; Johnson et al. 1999; Lima \& Gascon 1999; Lima \& Zakia 2000; Kageyama \& Gandara 2000). Porém, mesmo protegidas por legislação ambiental específica, estas formações foram e continuam sendo alteradas, principalmente por atividades antrópicas. As atividades agropecuárias associadas ao uso de queimadas e extrativismo florestal são apontadas como as principais causas da fragmentação florestal e degradação dos ecossistemas associados às bacias hidrográficas (Paine \& Ribic 2002; Corbacho et al. 2003).

O rio da Prata localizado na região sudoeste do Estado de Mato Grosso do Sul é considerado de rara beleza cênica e de importância ecológica. As nascentes deste rio estão inseridas no Planalto da Bodoquena, caracterizado por um maciço rochoso calcário elevado com altitudes que variam de 450 a $650 \mathrm{~m}$ (Boggiani 1999), onde se encontra um dos últimos remanescentes de floresta estacional semidecidual e decidual de grande extensão, com sua qualidade preservada (Pott \& Pott 2003). Esta região que compreende os municípios de Bonito, Bodoquena e parte de Jardim e Porto Murtinho tem sido intensamente explorada pelo turismo e, conseqüentemente, tem levado a um aumento substancial do interesse pela preservação e conservação dos recursos naturais. No entanto, vários trechos das formações ciliares associadas ao rio da Prata e de seus principais afluentes, rio Verde e córrego Beija-Flor, foram drasticamente eliminadas em função de atividades de exploração de madeira, implantação de atividades voltadas ao ecoturismo e, principalmente, pela expansão da agropecuária, levando à perda da biodiversidade local e colocando em risco a manutenção destes mananciais. As constantes ameaças e a escassez de estudos sobre a flora do Planalto da Bodoquena e áreas consideradas de entorno reforçam a necessidade urgente de levantamentos florísticos e fitossociológicos das espécies em trechos das matas ciliares do rio da Prata e em outros rios da região, visando subsidiar ações voltadas ao manejo, preservação e recomposição dessas formações. Nesse sentido, este estudo teve como objetivo analisar os aspectos fitossociológicos de um trecho da mata ciliar do rio da Prata no município de Jardim, Mato Grosso do Sul.

\section{Material e métodos}

Área de estudo - Este estudo foi desenvolvido em um trecho da mata ciliar do rio da Prata às margens da Serra da Bodoquena, no local denominado de "Santuário do Prata" ( $21^{\circ} 24^{\prime}$ 'S e $\left.56^{\circ} 22^{\prime} \mathrm{W}\right)$, a uma altitude média de 296m, município de Jardim (MS). De acordo com o Sistema Brasileiro de Classificação de Solos (EMBRAPA 1999), os solos predominantes na região de estudo são do tipo Argissolos, de textura arenosa média, profundos, monofásicos, não hidromórficos, apresentando fertilidade natural baixa e horizonte A moderado. O clima pode ser classificado segundo Köppen como Clima Tropical de Savana (Aw). A precipitação média anual para a região varia entre 1.400 a $1.600 \mathrm{~mm}$, sendo que o regime da precipitação é tipicamente tropical, apresentando dois períodos distintos: um chuvoso, que se inicia em outubro e estende-se até março, e outro seco, no período de abril a setembro. O mês com maior índice pluviométrico é janeiro, com uma precipitação média de 150 a $250 \mathrm{~mm}$. O mês mais seco é julho, com uma média de 10 a $40 \mathrm{~mm}$. A temperatura média anual varia entre $22{ }^{\circ} \mathrm{C}$ e $25^{\circ} \mathrm{C}$. O mês mais quente é outubro, com temperatura média entre $23^{\circ} \mathrm{C}$ e $27^{\circ} \mathrm{C}$, e o mais frio é julho, com temperatura variando de $17^{\circ} \mathrm{C}$ a $22^{\circ} \mathrm{C}$ (Campelo-Júnior et al. 1997). A micro-bacia do rio da Prata está inserida na sub-bacia hidrográfica do rio Miranda, que pertence à grande bacia hidrográfica do Rio Paraguai. Geologicamente, a região é caracterizada pela Formação Aquidauana - Bela Vista e pela Depressão do Miranda (Borges et al. 1997). Esta unidade constitui-se por terrenos baixos e relativamente planos, drenados pela sub-bacia do rio Miranda e encontra-se sob influência de formações florestais do tipo estacional decidual, estacional semidecidual e cerradão, também por formações savânicas e formações campestres como os campos secos e úmidos (Ribeiro \& Walter 1998). A principal atividade econômica desenvolvida na região é a agropecuária, formando extensas áreas campestres, dominada principalmente por espécies do gênero Brachiaria. As formações vegetais naturais apresentam-se fragmen- 
tadas, constituindo mosaicos de remanescentes florestais circundados por formações campestres nativas e exóticas, interligando as formações ciliares.

Aspectos gerais da vegetação - A faixa de mata ciliar amostrada tem aproximadamente 33,6 ha, compreendendo $2.240 \mathrm{~m}$ de extensão ao longo da margem do rio, e 150 a $200 \mathrm{~m}$ em direção à borda da mata. Perturbações antrópicas foram comuns na área de estudo, como a presença de trilhas em toda a extensão da margem, corte seletivo de madeira e pisoteio pelo rebanho bovino. A vegetação da margem sofre pouca influência do extravasamento do leito do rio na época das cheias, tendo em vista a altura do dique marginal que apresenta cerca de um a três metros de altura; porém, em certos locais da mata, ocorrem canais que permanecem úmidos durante a seca e inundados durante a cheia. A vegetação é densa, com dossel variando de 15 a $20 \mathrm{~m}$ em diferentes locais, sendo comum a presença de indivíduos emergentes com até cerca de $30 \mathrm{~m}$. Em determinados locais, ocorre a predominância das espécies Attalea phalerata Mart. ex Spreng. (acuri; bacuri) e Guadua paniculata Munro (taquara; bambu), formando agrupamentos homogêneos e densos. No estrato inferior ocorrem espécies como Psychotria carthagenensis Jacq. (Rubiaceae) e Clavija nutans (Vell.) Stahl (Teophrastaceae), além de grande número de plantas jovens de espécies arbóreas. É bastante frequiente a ocorrência de lianas, epífitas e grande diversidade de pteridófitas, principalmente no sub-bosque. Em determinados locais observam-se pequenas e médias clareiras ocasionadas pela queda natural de árvores e também pelo corte seletivo de espécies economicamente importantes como Myracrodruon urundeuva (Engl.) Fr. All. (aroeira) e Astronium graveolens Jacq. (gonçalo-alves) ocorrido num passado recente.

Amostragem - Para a amostragem da vegetação foi empregado o método de parcelas com distribuição contínua ao longo de seis transectos de 150 m cada. Estes transectos foram dispostos a intervalos aleatórios ao longo do rio e alinhados perpendicularmente à margem do rio, atravessando da margem à borda da floresta. Foram alocadas 15 parcelas de $10 \times 10 \mathrm{~m}$ ao longo de cada transecto, totalizando $9.000 \mathrm{~m}^{2}$ de área amostrada (0,9 ha). A distribuição das parcelas ao longo dos transectos procurou amostrar todas as variações presumidas para a composição e abundância das espécies a partir da margem do rio em direção à borda da mata. Os dados foram coletados mensalmente entre o período de agosto/2002 a julho/2003, sendo amostrados todos os indivíduos arbóreos-arbustivos vivos (exceto as espécies $A$. phalerata e G. paniculata) com perímetro a $1,30 \mathrm{~m}$ de altura do solo (PAP) igual ou superior a $10 \mathrm{~cm}$, o que corresponde a um diâmetro (DAP) de $\geq 3,18 \mathrm{~cm}$. Indivíduos ramificados abaixo dessa altura somente foram amostrados se pelo menos um dos ramos apresentasse o PAP mínimo estabelecido, sendo os demais medidos separadamente e somados ao de maior perímetro para cálculo da área basal. A suficiência amostral foi verificada pela análise gráfica da curva do coletor. Cada indivíduo amostrado foi marcado e numerado, utilizando-se etiquetas de aço inoxidável e teve altura máxima estimada, pela comparação com vara de coleta de $4 \mathrm{~m}$. O material botânico (reprodutivo e/ou vegetativo) foi prensado e herborizado pelos procedimentos usuais e identificado com auxílio de literatura especializada e comparações com exsicatas depositadas no Herbário (CGMS) da Universidade Federal de Mato Grosso do Sul, Campo Grande. Parte do material coletado encontra-se depositado no Herbário desta mesma Instituição.

As espécies foram categorizadas com relação à estratificação como pertencentes ao estrato superior (dossel e emergentes) e inferior (sub-bosque), baseado em observações pessoais e informações biológicas das espécies (Pott \& Pott 1994; Lorenzi 1998; 2000; Carmo \& Morellato 2000). Os parâmetros fitossociológicos estimados foram riqueza florística, diversidade, densidade absoluta (DA), densidade relativa (DR), dominância absoluta (DoA), dominância relativa (DoR), freqüência absoluta (FA), freqüência relativa (FR), índice do valor de importância (IVI) e de cobertura (IVC). O cálculo dos parâmetros fitossociológicos foi baseado em Whittaker (1972) por meio do programa FITOPAC (Shepherd 1996). Foram verificados os índices de diversidade de Shannon (H') e de eqüabilidade de Pielou (J') em base logarítmica neperiana (Magurram 1988).

\section{Resultados e discussão}

No total foram amostrados 661 indivíduos, pertencentes a 33 famílias botânicas, 57 gêneros e 66 espécies arbustivo-arbóreas (Tab. 1). Das 66 espécies, quatro estão identificadas apenas como gênero e uma como família. A curva do coletor mostrou uma tendência à estabilização de acréscimo de espécies com o número de parcelas amostradas (Fig. 1). Das 66 espécies registradas, 62 apresentaram hábito 
Tabela 1. Relação das famílias e espécies arbustivo-arbóreas ( $\mathrm{PAP} \geq 10 \mathrm{~cm}=\mathrm{DAP} \geq 3,18 \mathrm{~cm}$ ) ocorrentes em trecho de mata ciliar do Rio da Prata, município de Jardim, MS, Brasil, com seus nomes populares, hábito (A = árvore; B = arbusto) e estrato de ocorrência ( $\mathrm{I}=$ estrato inferior; $\mathrm{S}$ = estrato superior), e respectivos registros de herbário.

\begin{tabular}{lcc}
\hline Famílias/Espécies & Nomes populares & Hábito/Estrato Registro Herbário \\
CGMS
\end{tabular}

\begin{tabular}{|c|c|c|c|}
\hline \multicolumn{4}{|l|}{ ANACARDIACEAE } \\
\hline Astronium graveolens Jacq. & guaritá; gonçalo-alves & AS & 12928 \\
\hline Tapirira guianensis Aubl. & peito-de-pombo & AS & 12929 \\
\hline \multicolumn{4}{|l|}{ ANNONACEAE } \\
\hline Rollinia emarginata Schltdl. & ariticum-do-mato & AI & 12930 \\
\hline Unonopsis lindmanii R.E. Fr. & pindaíva-do-brejo & AI & 12931 \\
\hline \multicolumn{4}{|l|}{ APOCYNACEAE } \\
\hline Aspidosperma parvifolium A. DC. & guatambu-amarelo & AS & 12958 \\
\hline A. pyrifolium Mart. & peroba-branca & AS & 12932 \\
\hline \multicolumn{4}{|l|}{ BIGNONIACEAE } \\
\hline Tabebuia impetiginosa (Mart. ex Dc.) Standl. & piúva-da-mata & AS & 12933 \\
\hline T. roseo-alba (Ridl.) Sand. & ipê-branco & AS & 12934 \\
\hline \multicolumn{4}{|l|}{ BORAGINACEAE } \\
\hline Cordia sellowiana Cham. & louro-preto & AS & 12935 \\
\hline Patagonula americana $\mathrm{L}$. & guajuvira & AS & 12971 \\
\hline \multicolumn{4}{|l|}{ CAESALPINACEAE } \\
\hline Guibourtia hymenifolia (Moric.) J. Leonard. & jatobá-mirim & AS & 12941 \\
\hline Pterogyne nitens Tul. & amendoim-do-cerrado & AS & - \\
\hline \multicolumn{4}{|l|}{ CAPPARIDACEAE } \\
\hline Capparis retusa Gris & - & AI & - \\
\hline \multicolumn{4}{|l|}{ CARICACEAE } \\
\hline Jacaratia spinosa (Aubl.) A. DC. & mamão-do-mato & AS & 12951 \\
\hline \multicolumn{4}{|l|}{ CECROPIACEAE } \\
\hline Cecropia pachystachya Trécul. & embaúba & AS & 12948 \\
\hline \multicolumn{4}{|l|}{ COMBRETACEAE } \\
\hline Combretum leprosum Mart. & carne-de-vaca & AS & 12949 \\
\hline \multicolumn{4}{|l|}{ EUPHORBIACEAE } \\
\hline Adelia membranifolia (Müll. Arg.) Chodat \& Hassl. & espinheiro & AI & 12947 \\
\hline Croton urucurana Baill. & sangra-d'água & $\mathrm{AS}$ & 12927 \\
\hline Sapium haematospermum Müll. Arg. & leiteira & AS & 12926 \\
\hline \multicolumn{4}{|l|}{ FABACEAE } \\
\hline Dalbergia sp. & angelim-branco & AS & 12940 \\
\hline Holocalyx balansae Micheli & alecrim-de-campinas & AS & 12936 \\
\hline Myroxylon peruiferum L.f. & bálsamo & AS & 12950 \\
\hline \multicolumn{4}{|l|}{ FLACOURTIACEAE } \\
\hline Casearia decandra Jacq. & cafezeiro-do-mato & AI & 12945 \\
\hline C. sylvestris $\mathrm{Sw}$. & - & AI & 12946 \\
\hline \multicolumn{4}{|l|}{ GUTTIFERAE } \\
\hline Calophyllum brasilienses Camb. & guanandi & AS & - \\
\hline \multicolumn{4}{|l|}{ HIPPOCRATEACEAE } \\
\hline Salacia elliptica (Mart. ex Schult.) G. Don & saputã & AI & 12944 \\
\hline \multicolumn{4}{|l|}{ LAURACEAE } \\
\hline Nectandra membranacea (Swartz) Griseb. & canela-branca & AS & 12943 \\
\hline \multicolumn{4}{|l|}{ MALVACEAE } \\
\hline Bastardiopsis densiflora (Hook. \& Arn.) Hassl. & vassourão & AS & 12942 \\
\hline \multicolumn{4}{|l|}{ MELIACEAE } \\
\hline Cedrela fissilis Vell. & cedro-rosa & AS & - \\
\hline Guarea guidonea (L.) Sleumer & marinheiro & AS & 12957 \\
\hline G. kunthiana A. Juss. & canjambo & AI & 12955 \\
\hline Trichilia claussenii C. DC. & catiguá-vermelho & AI & 12956 \\
\hline T. hirta L. & cachuá & AI & 12953 \\
\hline T. pallida Sw. & catiguá & AI & 12954 \\
\hline T. silvatica C. DC. & - & AI & 12980 \\
\hline
\end{tabular}


Tabela 1 (continuação)

\begin{tabular}{|c|c|c|c|}
\hline Famílias/Espécies & Nomes populares & Hábito/Estrato & $\begin{array}{c}\text { Registro Herbário } \\
\text { CGMS }\end{array}$ \\
\hline \multicolumn{4}{|l|}{ MIMOSACEAE } \\
\hline Trichilia sp. & - & AI & 12952 \\
\hline Albizia hassleri (Chodat) Burkart. & angico-branco & AS & - \\
\hline Anadenanthera macrocarpa (Benth.) Brenan & angico-vermelho & AS & 12938 \\
\hline Inga marginata Willd. & ingá-do-brejo & AS & 12937 \\
\hline Parapiptadenia rigida (Benth.) Brenan & angico-da-mata & AS & 12939 \\
\hline \multicolumn{4}{|l|}{ MORACEAE } \\
\hline Ficus enormis (Mart. ex Miq.) Mart. & figueira-mata-pau & AS & - \\
\hline Maclura tinctoria (L.) D. Don ex Steud & amora-do-mato, taíuva & AS & 12979 \\
\hline \multicolumn{4}{|l|}{ MYRSINACEAE } \\
\hline Ardisia sp. & - & AI & - \\
\hline Myrsine umbellata Mart. & capororocão & AS & - \\
\hline \multicolumn{4}{|l|}{ MYRTACEAE } \\
\hline Campomanesia guazumifolia (Cambess.) O. Berg & sete-capotes & AI & 12977 \\
\hline Myrcianthes pungens (O. Berg) D. Legrand. & guabiroba & AS & 12978 \\
\hline Eugenia florida DC. & jamelão-do-campo & AI & 12976 \\
\hline \multicolumn{4}{|l|}{ NYCTAGINACEAE } \\
\hline Guapira opposita (Vell.) Reitz. & maria-mole & AI & 12975 \\
\hline \multicolumn{4}{|l|}{ OLACACEAE } \\
\hline Ximenia americana $\mathrm{L}$. & limão-do-mato & AI & 12970 \\
\hline \multicolumn{4}{|l|}{ OPILIACEAE } \\
\hline Agonandra brasiliensis Miers ex Benth. \& Hook.f. & tinge-cuia & AS & 12972 \\
\hline \multicolumn{4}{|l|}{ PIPERACEAE } \\
\hline Piper angustifolium Lam. & pimenta-do-mato & BI & 12959 \\
\hline P. tuberculatum Jacq. & pimenta-do-mato & BI & 12973 \\
\hline \multicolumn{4}{|l|}{ RHAMNACEAE } \\
\hline Rhamnidium elaeocarpum Reissek & cabriteira & AS & 12969 \\
\hline \multicolumn{4}{|l|}{ RUBIACEAE } \\
\hline Alibertia sessilis Schumann & marmelo-do-campo & AI & 12968 \\
\hline Psychotria carthagenensis Jacq. & & BI & 12967 \\
\hline \multicolumn{4}{|l|}{ RUTACEAE } \\
\hline Helietta apiculata Benth. & canela-de-cutia & AS & 12974 \\
\hline \multicolumn{4}{|l|}{ RUTACEAE } \\
\hline Zanthoxylum hasslerianum (Chodat) Pirani & mamica-de-porca & AI & 12981 \\
\hline Rutaceae sp. 1 & - & AI & - \\
\hline \multicolumn{4}{|l|}{ SAPINDACEAE } \\
\hline Allophyllus edulis (St. Hil.) Radlk. & vacum & AI & 12964 \\
\hline Cupania castaneaefolia Mart. & camboatá & AI & - \\
\hline Averrhoidium paraguaiense Radlk. & maria-preta & AS & 12966 \\
\hline Talisia esculenta (A. St.-Hil.) Radlk. & pitomba & AS & 12965 \\
\hline \multicolumn{4}{|l|}{ SAPOTACEAE } \\
\hline Chrysophyllum gonocarpum (Mart. \& Eichl.) Engl. & aguaí-da-serra & AS & 12963 \\
\hline \multicolumn{4}{|l|}{ STERCULIACEAE } \\
\hline Guazuma ulmifolia Lam. & chico-magro & AS & 12962 \\
\hline \multicolumn{4}{|l|}{ ULMACEAE } \\
\hline Celtis pubescens Spreng. & taleira, esporão-de-galo & AI & 12961 \\
\hline Trema micrantha (L.) Blume & piriquiteira & AI & 12960 \\
\hline
\end{tabular}

arbóreo sendo que $38,70 \%$ foram consideradas como pertencentes ao estrato inferior e $61,30 \%$ como tipicamente do estrato superior (dossel ou emergentes). Apenas quatro espécies apresentaram hábito arbustivo, Ardisia sp., Piper angustifolium, P. tuberculatum e Psychotria carthagenensis (Tab. 1).

As sete famílias de maior riqueza florística contribuíram com $42,42 \%$ das espécies amostradas, e as demais 26 famílias com 57,58\% das espécies. A família Meliaceae, representada por oito espécies, foi a de maior riqueza, perfazendo $12,12 \%$ do total de espécies registradas. As famílias Mimosaceae e Sapindaceae foram representadas com quatro espécies cada $(6,06 \%$ cada), seguidas pelas famílias, Euphorbiaceae, Fabaceae, Myrtaceae e Rutaceae, que apresentaram três espécies cada $(4,55 \%$ cada). 


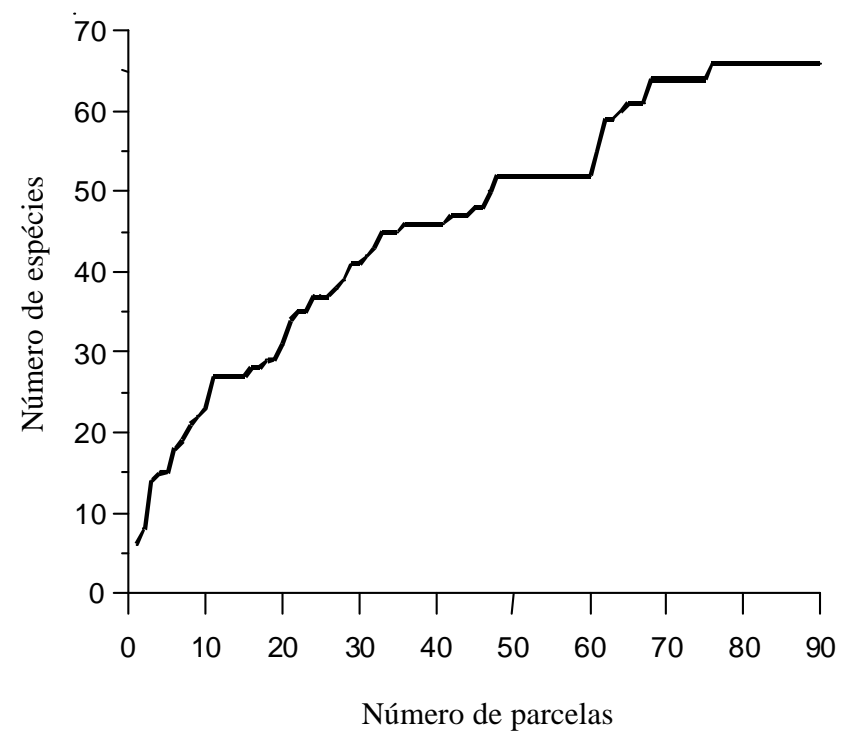

Figura 1. Curva do coletor para as espécies amostradas em trecho da mata ciliar do rio da Prata no município de Jardim, MS, Brasil.

Doze famílias $(36,36 \%)$ foram representadas por duas espécies e 14 famílias $(21,21 \%)$ por somente uma espécie. Os gêneros que mais contribuíram com o número de espécies foram Trichilia, com cinco espécies, e Aspidosperma, Casearia, Guarea e Tabebuia, com duas cada (Tab. 1). Estes resultados são semelhantes aos obtidos por Leitão-Filho (1982), Salis et al. (1994), Rodrigues \& Nave (2000) e Felfili et al. (2001) que citam as famílias Caesalpinaceae, Euphorbiaceae, Fabaceae, Meliaceae, Mimosaceae, Myrtaceae e Rutaceae como as mais representativas em número de espécies arbustivo-arbóreas nas matas ciliares. Além destas famílias, foram registradas neste estudo as famílias Anacardiaceae, Annonaceae e Bignoniaceae, também consideradas importantes nas formações ciliares por Silva Junior et al. (2001b). Se tivéssemos considerado Caesalpiniaceae, Mimosaceae e Fabaceae como uma única família, Leguminosae, esta passaria a ser a de maior riqueza florística, com nove espécies para a área amostrada, corroborando com os autores acima citados, que descrevem a importância desta família para as matas ciliares. Leitão-Filho (1982), descrevendo a estrutura das matas ciliares, relata que nos estratos superiores existe clara dominância de Leguminosae e que em nenhuma outra formação florestal do Estado de São Paulo, a dominância de Leguminosae é tão alta como ao longo destas formações, principalmente por mimosáceas.

O índice de diversidade de Shannon encontrado para a área total foi de $\mathrm{H}^{\prime}=3,413$ nats/indivíduo e a eqüabilidade de Pielou foi J'=0,815. Considerando o tamanho da área amostral ( 0,9 ha) e o diâmetro mínimo estabelecido (DAP $\geq 3,18 \mathrm{~cm}$ ), esperava-se encontrar para o trecho da mata ciliar do Rio da Prata número de espécies maior do que o amostrado, segundo a alta riqueza florística, entre 247 espécies arbustivo-arbóreas descrita para as formações ciliares (Rodrigues \& Nave 2000). No entanto, o valor da diversidade de Shannon foi semelhante ao encontrado em outras localidades (Tab. 2). A eqüabilidade igual a 0,815 encontrada para o trecho de mata ciliar estudado foi alta, indicando que as populações das espécies ocorrentes nessa mata apresentam distribuição quase homogênea, sugerindo estabilidade na abundância destas espécies. Esta homogeneidade na abundância das espécies contribuiu para elevar o valor do índice de diversidade, uma vez que o número de espécies registradas na área de estudo foi relativamente baixo. A menor riqueza florística e diversidade em espécies encontradas neste estudo, quando comparado com estudos conduzidos na Região Sudeste, pode estar relacionada a fatores físicos locais e a processos de fragmentação e perturbações que propiciaram condições para dominância de algumas espécies no trecho estudado. Ao longo de toda a área estudada foram observados elevada abundância de A. phalerata (Palmae) e freqüentes agrupamentos de G. paniculata (Gramineae). Estas espécies predominam em determinados locais devido principalmente às características edáficas, e topográficas e, possivelmente, como resultado de perturbações sofridas em passado recente, pois segundo Pott \& Pott (2003) a espécie A. phalerata é típica de formações secundárias. Estudos complementares em outros trechos de matas ciliares, com as mesmas características físicas da área estudada seriam importantes para possibilitar comparações quanto à riqueza florística, uma vez que este estudo mostra os primeiros resultados que descrevem e avaliam a composição florística para essa região.

As famílias Meliaceae, Myrtaceae, Euphorbiaceae, Annonaceae, Fabaceae, Mimosaceae, Rutaceae, Caesalpiniaceae, Ulmaceae e Sapindaceae representaram $79,58 \%$ do total de indivíduos registrados. As demais famílias contribuíram com 20,42\% dos indivíduos. A família Meliaceae foi a mais representativa, com $17,85 \%$ do total dos indivíduos amostrados, destacando-se os gêneros Guarea e Trichilia considerados típicos de formações ciliares. A família Myrtaceae foi fortemente representada pela espécie Myrcianthes pungens que contribuiu com $13,46 \%$ do total dos indivíduos. Segundo Romagnolo 
Tabela 2. Relação da riqueza florística e diversidade de espécies arbóreas e arbustivas, por área amostral em formações florestais na região Sudeste e Centro-Oeste do Brasil. H' = índice de diversidade de Shannon.

\begin{tabular}{|c|c|c|c|c|c|}
\hline Tipo de vegetação & Área amostral & DAP & Riqueza & $\begin{array}{c}\text { Diversidade } \\
\left(\mathrm{H}^{\prime}\right)\end{array}$ & Referências \\
\hline Floresta estacional semidecidual, rio Ingaí, MG & 1 ha & $\geq 5 \mathrm{~cm}$ & 140 & 3,734 & Botrel et al. 2002 \\
\hline Floresta estacional semidecidual, rio Passa Cinco, SP & P 0,785 ha & $\geq 5 \mathrm{~cm}$ & 107 & 3,60 & Bertani et al. 2001 \\
\hline Floresta ripária, rio Paraná, MS & 0,945 ha & $\geq 4,77 \mathrm{~cm}$ & 58 & 3,20 & Romagnolo \& Souza (2000) \\
\hline Mata ciliar, rio da Prata, MS & 0,9 ha & $\geq 3,18 \mathrm{~cm}$ & 66 & 3,413 & Este estudo \\
\hline
\end{tabular}

\& Souza (2004) a família Myrtaceae assume grande importância ecológica nas diferentes formações vegetacionais do Brasil, como em ambientes ripários, incluindo a planície alagável do alto rio Paraná.

As 10 espécies de maior densidade perfazem $61,57 \%$ do total de indivíduos amostrados (Tab. 3). As espécies Guarea kunthiana, Unonopsis lindmanii, Trichilia claussenii, Holocalyx balansae e o gênero Dalbergia são citados por Rodrigues \& Nave (2000) como pertencentes as florestas ciliares do Brasil extraamazônico e por Felfili et al. (2001) para as matas de galeria e ciliares do Bioma Cerrado. M. pungens é citada por Silva Júnior et al. (1998, 2001b) e por Felfili et al. (2001) como sendo comum em vegetação ciliar. Helietta apiculata e Celtis pubescens também ocorrem em formações ciliares (Rodrigues \& Nave 2000). Somente para Adelia membranifolia e Averrhoidium paraguaiense não foram encontrados registros de ocorrência em matas ciliares. A. membranifolia, a segunda mais representativa em número de indivíduos, ocorre com freqüência nas clareiras e bordas da área estudada. U. lindmanii é considerada espécie ombrófila típica das matas ciliares do Brasil Central (Oliveira Filho \& Ratter 2000), com elevado índice de valor de importância no trabalho realizado por Romagnolo \& Souza (2000). Verificou-se para o trecho de mata ciliar do rio da Prata uma composição florística mista com espécies típicas destas formações, como Cecropia pachystachya, Croton urucurana, Guarea kunthiana, G. guidonea, Inga marginata, Tapirira guianensis, Trichilia claussenii e Unonopsis lindmanii e também espécies freqüentes em florestas estacionais semidecíduas e decíduas, como Albizia hassleri, Anadenanthera macrocarpa, Aspidosperma parvifolium, A. pyrifolium, Astronium graveolens, Cedrela fissilis, Parapiptadenia rigida e Tabebuia impetiginosa. Estes resultados permitem inferir a presença de uma floresta estacional semidecídua associada ao rio da Prata com forte influência na composição florística local.
Dezessete espécies $(25,76 \%)$ foram representadas por apenas um indivíduo e cinco $(7,58 \%)$ por dois indivíduos (Tab. 3), perfazendo $4,08 \%$ do total de indivíduos registrados. Em função da alta riqueza de espécies arbóreas da maioria das florestas tropicais, é muito comum em estudos fitossociológicos a ocorrência de número elevado de espécies representadas por um ou poucos indivíduos dentro da área amostral. Por exemplo, Carvalho et al. (2000) amostraram $24,87 \%$ do total das espécies com apenas um indivíduo e Botrel et al. (2002) encontraram $28,57 \%$ de espécies consideradas raras. O número de espécies com um único indivíduo na área estudada é semelhante, quando comparado com os obtidos por estes autores; no entanto, isto não significa que tais espécies sejam raras, mas que, na maior parte das vezes, apresentam distribuição espacial que determina a ocorrência de poucos indivíduos por unidade de área (Pagano et al. 1995; Kageyama \& Gandara 1998; Ivanauskas et al. 1999). Segundo Borém \& OliveiraFilho (2002) quanto maior a riqueza em espécies arbóreas e mais avançado o estádio de maturação de uma comunidade vegetal maior é o número de espécies consideras raras.

O total de indivíduos (661) amostrados na área de (0,9 ha) corresponderam a $19,19 \mathrm{~m}^{2}$ de área basal. A densidade específica e a área basal estimada para (1 ha) foi de 734,44 indivíduos/ha e $21,32 \mathrm{~m} / \mathrm{ha}$, respectivamente. Estes valores são muito baixos quando comparados com outros estudos conduzidos em outras formações ciliares (Carvalho et al. 2000; Bertani et al. 2001). Por exemplo, Botrel et al. (2002), encontraram 2.683 indíviduos/ha e uma área basal de $29,309 \mathrm{~m}^{2} /$ ha para uma comunidade arbóreo-arbustiva de uma floresta estacional semidecidual ribeirinha em Ingaí, MG. Sampaio et al. (2000), estudando a diversidade e distribuição de espécies arbóreas (DAP $\geq 5 \mathrm{~cm}$ ) em duas matas de galeria na micro-bacia do Riacho Fundo, DF, amostraram 1.333 indíviduos/ha e uma área basal de $34,4 \mathrm{~m}^{2} / \mathrm{ha}$ para trechos não-inundáveis e 
Tabela 3. Parâmetros fitossociológicos das espécies arbóreas-arbustivas amostradas em trecho da mata ciliar do Rio da Prata, município de Jardim, MS, Brasil. N: Número de indivíduos; FA: Freqüência Absoluta (\%) FR: Frequiência Relativa (\%); DA: Densidade Absoluta (n.ind/ha); DR: Densidade Relativa (\%); DoA: Dominância Absoluta (m²/ha); DoR: Dominância Relativa (\%); IVI: Índice de Valor de Importância; IVC: Índice do Valor de Cobertura; Amáx: Altura máxima (m); Dmáx: Diâmetro máximo (cm). Espécies relacionadas em seqüência decrescente de IVI.

\begin{tabular}{|c|c|c|c|c|c|c|c|c|c|c|c|}
\hline Espécies & $\mathrm{N}$ & FA & FR & DA & DR & DoA & DoR & IVI & IVC & Amáx & Dmáx \\
\hline Myrcianthes pungens & 89 & 46,67 & 9,74 & 98,89 & 13,46 & 1,118 & 5,24 & 28,45 & 18,71 & 20,0 & 39,43 \\
\hline Adelia membranifolia & 72 & 44,44 & 9,28 & 80,00 & 10,89 & 0,994 & 4,66 & 24,84 & 15,55 & 10,0 & 29,90 \\
\hline Dalbergia sp. & 38 & 18,89 & 3,94 & 42,22 & 5,75 & 2,296 & 10,77 & 20,46 & 16,51 & 21,0 & 52,47 \\
\hline Holocalyx balansae & 23 & 18,89 & 3,94 & 25,56 & 3,48 & 1,693 & 7,94 & 15,36 & 11,42 & 22,0 & 58,19 \\
\hline Unonopsis lindmanii & 43 & 28,89 & 6,03 & 47,78 & 6,51 & 0,382 & 1,79 & 14,33 & 8,30 & 12,0 & 21,62 \\
\hline Guarea kunthiana & 44 & 26,67 & 5,57 & 48,89 & 6,66 & 0,363 & 1,70 & 13,93 & 8,36 & 14,0 & 23,21 \\
\hline Trichilia claussenii & 33 & 24,44 & 5,10 & 36,67 & 4,99 & 0,795 & 3,73 & 13,83 & 8,72 & 15,0 & 29,26 \\
\hline Guarea guidonea & 16 & 13,33 & 2,78 & 17,78 & 2,42 & 0,871 & 4,08 & 9,29 & 6,51 & 17,0 & 43,88 \\
\hline Averrhoidium paraguaiense & 21 & 17,78 & 3,71 & 23,33 & 3,18 & 0,341 & 1,60 & 8,49 & 4,78 & 16,0 & 26,71 \\
\hline Astronium graveolens & 13 & 11,11 & 2,32 & 14,44 & 1,97 & 0,841 & 3,94 & 8,23 & 5,91 & 23,0 & 60,42 \\
\hline Celtis pubescens & 22 & 11,11 & 2,32 & 24,44 & 3,33 & 0,534 & 2,51 & 8,15 & 5,83 & 12,0 & 56,60 \\
\hline Parapiptadenia rigida & 7 & 6,67 & 1,39 & 7,78 & 1,06 & 1,207 & 5,66 & 8,11 & 6,72 & 27,0 & 95,40 \\
\hline Nectandra membranacea & 17 & 13,33 & 2,78 & 18,89 & 2,57 & 0,514 & 2,41 & 7,77 & 4,98 & 17,0 & 34,19 \\
\hline Myrsine umbellata & 8 & 8,89 & 1,86 & 8,89 & 1,21 & 0,844 & 3,96 & 7,02 & 5,17 & 22,0 & 48,65 \\
\hline Inga marginata & 19 & 16,67 & 3,48 & 21,11 & 2,87 & 0,093 & 0,44 & 6,79 & 3,31 & 10,0 & 20,99 \\
\hline Chrysophyllum gonocarpum & 13 & 11,11 & 2,32 & 14,44 & 1,97 & 0,510 & 2,39 & 6,68 & 4,36 & 17,0 & 34,98 \\
\hline Myroxylon peruiferum & 7 & 6,67 & 1,39 & 7,78 & 1,06 & 0,890 & 4,17 & 6,63 & 5,23 & 23,0 & 69,96 \\
\hline Helietta apiculata & 22 & 11,11 & 2,32 & 24,44 & 3,33 & 0,156 & 0,73 & 6,38 & 4,06 & 12,0 & 18,13 \\
\hline Jacaratia spinosa & 4 & 4,44 & 0,93 & 4,44 & 0,61 & 0,796 & 3,73 & 5,26 & 4,34 & 15,0 & 74,41 \\
\hline Tabebuia impetiginosa & 5 & 5,56 & 1,16 & 5,56 & 0,76 & 0,713 & 3,34 & 5,26 & 4,10 & 25,0 & 69,96 \\
\hline Albizia haslerii & 6 & 6,67 & 1,39 & 6,67 & 0,91 & 0,608 & 2,85 & 5,15 & 3,76 & 18,0 & 45,47 \\
\hline Combretum leprosum & 2 & 2,22 & 0,46 & 2,22 & 0,30 & 0,795 & 3,73 & 4,49 & 4,03 & 17,0 & 90,63 \\
\hline Guibourtia hymenifolia & 5 & 5,56 & 1,16 & 5,56 & 0,76 & 0,429 & 2,01 & 3,93 & 2,77 & 22,0 & 57,24 \\
\hline Aspidosperma parvifolium & 7 & 5,56 & 1,16 & 7,78 & 1,06 & 0,359 & 1,68 & 3,90 & 2,74 & 19,0 & 41,82 \\
\hline Trichilia sp. & 9 & 7,78 & 1,62 & 10,00 & 1,36 & 0,040 & 0,19 & 3,17 & 1,55 & 07,0 & 12,40 \\
\hline Salacia elliptica & 4 & 4,44 & 0,93 & 4,44 & 0,61 & 0,342 & 1,61 & 3,14 & 2,21 & 13,0 & 56,29 \\
\hline Maclura tinctoria & 4 & 4,44 & 0,93 & 4,44 & 0,61 & 0,331 & 1,55 & 3,09 & 2,16 & 18,0 & 54,06 \\
\hline Guapira opposita & 6 & 5,56 & 1,16 & 6,67 & 0,91 & 0,210 & 0,98 & 3,05 & 1,89 & 15,0 & 23,85 \\
\hline Aspidosperma pyrifolium & 7 & 5,56 & 1,16 & 7,78 & 1,06 & 0,087 & 0,41 & 2,63 & 1,47 & 12,0 & 20,03 \\
\hline Zanthoxylum hasslerianum & 7 & 6,67 & 1,39 & 7,78 & 1,06 & 0,028 & 0,13 & 2,58 & 1,19 & 08,0 & 09,54 \\
\hline Tabebuia roseo-alba & 4 & 3,33 & 0,70 & 4,44 & 0,61 & 0,271 & 1,27 & 2,57 & 1,88 & 12,0 & 43,88 \\
\hline Trichilia pallida & 8 & 4,44 & 0,93 & 8,89 & 1,21 & 0,073 & 0,34 & 2,48 & 1,55 & 10,0 & 20,03 \\
\hline Tapirira guianensis & 6 & 4,44 & 0,93 & 6,67 & 0,91 & 0,130 & 0,61 & 2,45 & 1,52 & 11,0 & 24,17 \\
\hline Eugenia florida & 7 & 5,56 & 1,16 & 7,78 & 1,06 & 0,013 & 0,06 & 2,28 & 1,12 & 07,0 & 06,20 \\
\hline Cedrela fissilis & 3 & 2,22 & 0,46 & 3,33 & 0,45 & 0,261 & 1,23 & 2,14 & 1,68 & 17,0 & 48,34 \\
\hline Ximenia americana & 2 & 2,22 & 0,46 & 2,22 & 0,30 & 0,255 & 1,19 & 1,96 & 1,50 & 12,0 & 50,88 \\
\hline Croton urucurana & 4 & 3,33 & 0,70 & 4,44 & 0,61 & 0,125 & 0,59 & 1,89 & 1,19 & 17,0 & 34,03 \\
\hline Campomanesia guazumifolia & 4 & 4,44 & 0,93 & 4,44 & 0,61 & 0,052 & 0,24 & 1,78 & 0,85 & 05,0 & 21,60 \\
\hline Patagonola americana & 3 & 3,33 & 0,70 & 3,33 & 0,45 & 0,132 & 0,62 & 1,77 & 1,08 & 23,0 & 38,48 \\
\hline Psychotria carthagenensis & 4 & 4,44 & 0,93 & 4,44 & 0,61 & 0,006 & 0,03 & 1,56 & 0,63 & 03,0 & 05,41 \\
\hline Casearia decandra & 4 & 2,22 & 0,46 & 4,44 & 0,61 & 0,089 & 0,42 & 1,48 & 1,02 & 06,0 & 28,00 \\
\hline Rhamnidium elaeocarpum & 3 & 3,33 & 0,70 & 3,33 & 0,45 & 0,065 & 0,30 & 1,45 & 0,76 & 10,0 & 26,71 \\
\hline Cecropia pachystachya & 4 & 2,22 & 0,46 & 4,44 & 0,61 & 0,055 & 0,26 & 1,33 & 0,86 & 08,0 & 16,85 \\
\hline Anadenanthera macrocarpa & 1 & 1,11 & 0,23 & 1,11 & 0,15 & 0,188 & 0,88 & 1,27 & 1,03 & 15,0 & 46,43 \\
\hline Guazuma ulmifolia & 3 & 2,22 & 0,46 & 3,33 & 0,45 & 0,015 & 0,07 & 0,99 & 0,52 & 10,0 & 09,54 \\
\hline Rollinia emarginata & 3 & 2,22 & 0,46 & 3,33 & 0,45 & 0,011 & 0,05 & 0,97 & 0,50 & 06,0 & 08,90 \\
\hline Trichilia hirta & 3 & 2,22 & 0,46 & 3,33 & 0,45 & 0,010 & 0,04 & 0,96 & 0,50 & 06,0 & 06,83 \\
\hline Cordia sellowiana & 1 & 1,11 & 0,23 & 1,11 & 0,15 & 0,115 & 0,54 & 0,92 & 0,69 & 15,0 & 36,25 \\
\hline Trichilia silvatica & 2 & 2,22 & 0,46 & 2,22 & 0,30 & 0,004 & 0,02 & 0,79 & 0,32 & 04,0 & 05,72 \\
\hline Ardisia sp. & 2 & 2,22 & 0,46 & 2,22 & 0,30 & 0,004 & 0,02 & 0,78 & 0,32 & 03,0 & 04,77 \\
\hline Indeterminada 1 & 2 & 2,22 & 0,46 & 2,22 & 0,30 & 0,002 & 0,01 & 0,78 & 0,31 & 03,0 & 03,81 \\
\hline Pterogyne nitens & 1 & 1,11 & 0,23 & 1,11 & 0,15 & 0,075 & 0,35 & 0,73 & 0,50 & 13,0 & 21,47 \\
\hline
\end{tabular}


Tabela 3 (continuação)

\begin{tabular}{|c|c|c|c|c|c|c|c|c|c|c|c|}
\hline Espécies & $\mathrm{N}$ & $\mathrm{FA}$ & FR & DA & $\mathrm{DR}$ & DoA & DoR & IVI & IVC & Amáx & Dmáx \\
\hline Sapium haematospermum & 1 & 1,11 & 0,23 & 1,11 & 0,15 & 0,040 & 0,19 & 0,57 & 0,34 & 12,0 & 19,56 \\
\hline Ficus enormis & 1 & 1,11 & 0,23 & 1,11 & 0,15 & 0,033 & 0,16 & 0,54 & 0,31 & 12,0 & 19,08 \\
\hline Talisia esculenta & 1 & 1,11 & 0,23 & 1,11 & 0,15 & 0,032 & 0,15 & 0,53 & 0,30 & 08,0 & 17,49 \\
\hline Capparis retusa & 1 & 1,11 & 0,23 & 1,11 & 0,15 & 0,027 & 0,13 & 0,51 & 0,28 & 08,0 & 17,49 \\
\hline Cupania castaneaefolia & 1 & 1,11 & 0,23 & 1,11 & 0,15 & 0,020 & 0,10 & 0,48 & 0,25 & 12,0 & 12,56 \\
\hline Alibertia sessilis & 1 & 1,11 & 0,23 & 1,11 & 0,15 & 0,014 & 0,06 & 0,45 & 0,22 & 05,0 & 08,59 \\
\hline Agonandra brasiliensis & 1 & 1,11 & 0,23 & 1,11 & 0,15 & 0,006 & 0,03 & 0,41 & 0,18 & 05,0 & 07,95 \\
\hline Casearia sylvestris & 1 & 1,11 & 0,23 & 1,11 & 0,15 & 0,006 & 0,03 & 0,41 & 0,18 & 07,0 & 07,31 \\
\hline Bastardiopsis densiflora & 1 & 1,11 & 0,23 & 1,11 & 0,15 & 0,004 & 0,02 & 0,40 & 0,17 & 05,0 & 05,72 \\
\hline Piper tuberculatum & 1 & 1,11 & 0,23 & 1,11 & 0,15 & 0,004 & 0,02 & 0,40 & 0,17 & 02,0 & 06,36 \\
\hline Trema micrantha & 1 & 1,11 & 0,23 & 1,11 & 0,15 & 0,004 & 0,02 & 0,40 & 0,17 & 04,0 & 03,81 \\
\hline Allophylus edulis & 1 & 1,11 & 0,23 & 1,11 & 0,15 & 0,003 & 0,01 & 0,40 & 0,16 & 04,0 & 05,72 \\
\hline Calophyllum brasilienses & 1 & 1,11 & 0,23 & 1,11 & 0,15 & 0,001 & 0,01 & 0,39 & 0,16 & 01,5 & 03,81 \\
\hline Piper angustifolium & 1 & 1,11 & 0,23 & 1,11 & 0,15 & 0,001 & 0,01 & 0,39 & 0,16 & 03,0 & 03,49 \\
\hline
\end{tabular}

2.094 indíviduos/ha e área basal 33,3 $\mathrm{m}^{2} / \mathrm{ha}$ para inundáveis. As condições edáficas locais, juntamente com o histórico de perturbações naturais e antrópicas, podem estar contribuindo para a baixa abundância de indivíduos na área amostrada. Porém, são necessários estudos complementares que possam avaliar a possível interferência de fatores antrópicos no recrutamento e estabelecimento de indivíduos arbóreo-arbustivos para as formações ciliares do rio da Prata.

A análise dos parâmetros fitossociológicos mostrou que as 10 espécies de maior índice de valor de importância (IVI) perfizeram 52,4\% deste índice e as dez espécies de maior índice de valor de cobertura (IVC) corresponderam a 53,35\% deste índice (Tab. 3). As espécies Myrcianthes pungens e Adelia membranifolia destacaram-se nesta comunidade por apresentarem a combinação de grande número de indivíduos e altos valores de frequiência nas parcelas, além de alta dominância relativa, mostrando serem espécies numerosas e com ampla distribuição na área de estudo. Dalbergia sp., Holocalyx balansae, Astronium graveolens, Guarea guidonea e Parapiptadenia rigida, apesar de estarem representadas por menos indivíduos, também apresentaram elevados IVIs e IVCs, devido ao elevado diâmetro de seus indivíduos, que influenciou a dominância relativa (DoR). Unonopsis lindmanii, Trichilia claussenii, Guarea kunthiana apresentaram valores baixos para dominância relativa, porém ocorreram com altas freqüências e densidades relativas, elevando seus valores de importância e cobertura. Dentre as espécies com elevados IVIs, A. graveolens, A. membranifolia, $H$. balansae e $U$. lindmanii, foram amostradas por
Damasceno Júnior et al. (2000) em levantamento florístico realizado nas cabeceiras do rio da Prata, demonstrando serem espécies comuns para a região de estudo.

A distribuição da freqüência de árvores por classes de altura para a comunidade mostrou que $39,03 \%$ das árvores amostradas apresentaram alturas entre 1,5 a $5 \mathrm{~m}$, e $37,07 \%$ estão entre 6 a $10 \mathrm{~m}$ (Fig. 2). Este grande número de indivíduos $(76,10 \%)$ nestas duas primeiras classes de altura indica um número elevado de árvores jovens típicas do dossel ou emergentes que estão ocupando o estrato inferior, uma vez que $61,29 \%$ das espécies amostradas foram consideradas como características do estrato superior (Tab. 1). O dossel ou estrato superior que compreende alturas entre $11 \mathrm{a}$ $20 \mathrm{~m}$, foi representado por $21,94 \%$ das árvores e somente $1,97 \%$, destacaram-se como emergentes. Em relação a distribuição dos indivíduos em classes de diâmetro verificou-se que $51,13 \%$ do total de indivíduos amostrados apresentaram diâmetros entre 3,18 a $10 \mathrm{~cm}$, e $26,93 \%$ entre 10,1 a $20 \mathrm{~cm}$ (Fig. 3). Estes resultados indicam predominância no estrato inferior de árvores baixas e finas, como Adelia membranifolia, Celtis pubescens, Guarea kunthiana, Helietta apiculata, Inga marginata, Trichilia claussenii e Unonopsis lindmanii. As espécies Astronium graveolens, Chrysophyllum gonocarpum, Cedrela fissilis, Combretum leprosum, Guarea guidonea, Jacaratia spinosa, Parapiptadenia rigida, Tabebuia impetiginosa, Salacia elliptica, Guibourtia hymenifolia, Maclura tinctoria, Ximenia americana e Anadenanthera macrocarpa apresentaram diâmetros superiores a $40 \mathrm{~cm}$ e ocuparam o dossel ou 


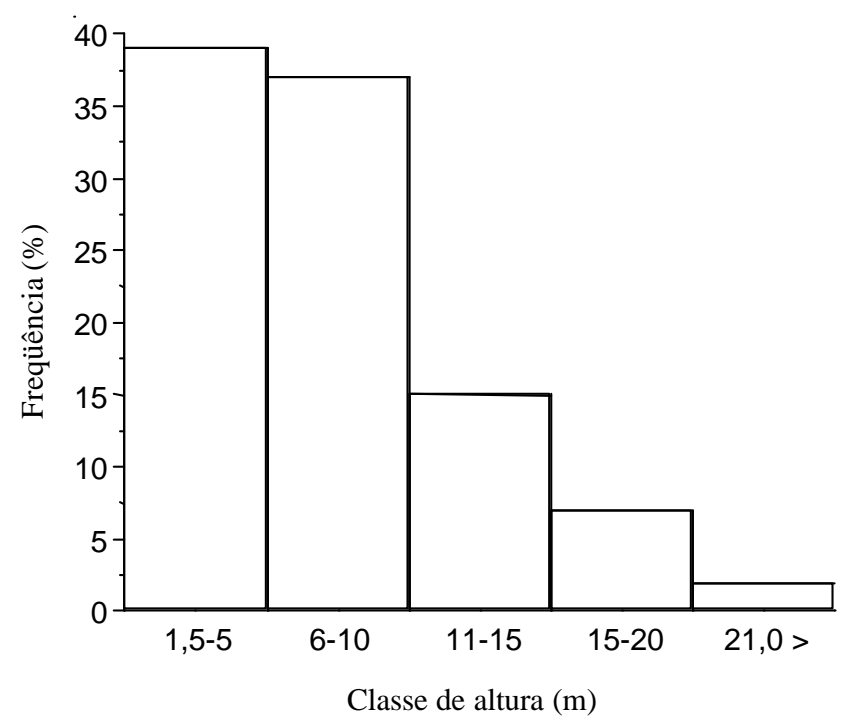

Figura 2. Distribuição da freqüência relativa de indivíduos em classes de altura em trecho da mata ciliar do rio da Prata, município de Jardim, MS, Brasil.

destacaram-se como emergentes na área estudada. Segundo Nunes et al. (2003), áreas que sofreram perturbações mais severas no passado possuem maiores densidades de árvores finas e baixas, caracterizando estádio de regeneração inicial, já setores que sofreram distúrbios mais leves no passado apresentam maior densidade de árvores altas e grossas, indicando estádio regenerativo mais avançado. Apesar do trecho de mata ciliar estudado apresentar predominância de árvores baixas e finas, destacaram-se espécies e indivíduos com elevada altura e diâmetro, sugerindo uma comunidade estruturada em fase de regeneração avançada, uma vez que espécies típicas de formações secundárias, como C. pachystachya, C. urucurana, T. guianensis e A. macrocarpa apresentaram baixas densidades na área estudada.

As formações florestais associadas a cursos d'água, caracterizadas como áreas de preservação permanente são amplamente protegidas pela Legislação Ambiental Brasileira (Lei n. 4.771/65, alterada pela Lei n. 7.803/89) que estabelece exigências diferenciadas para a cobertura vegetal destinada a proteger nascentes, margens de rios, córregos, lagos e reservatórios de água. Os resultados obtidos neste estudo, como riqueza florística, diversidade, composição e abundância das espécies corroboram e reforçam a importância da Legislação Ambiental Federal e Estadual, Lei n. 1.871 de 15/07/1998 (Morelli 2000) na manutenção e preservação da faixa ciliar para as margens do rio da Prata. Também, são de extrema relevância para subsidiar ações e projetos que visem o

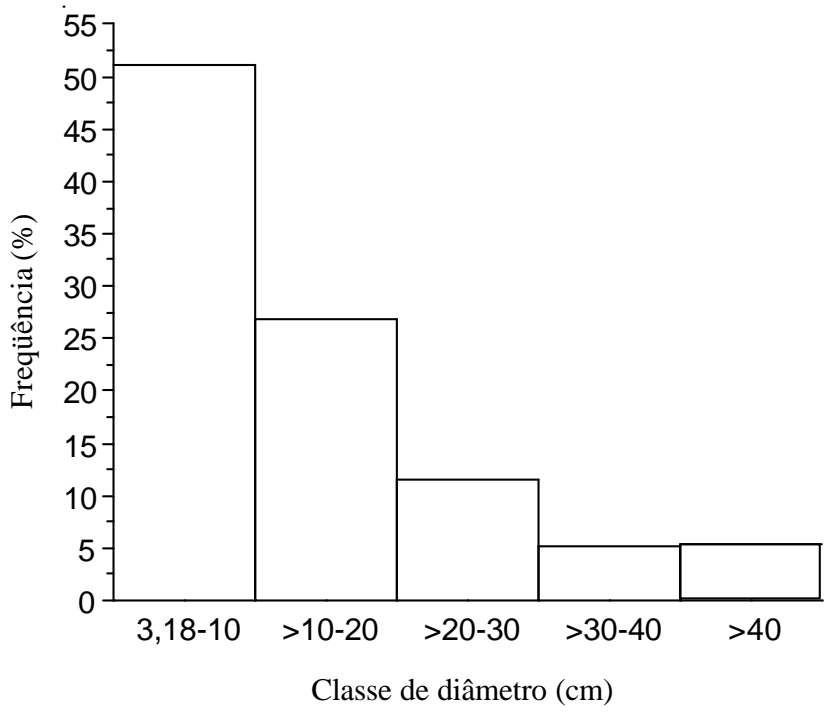

Figura 3. Distribuição da freqüência relativa de indivíduos em classes de diâmetro em trecho da mata ciliar do rio da Prata, município de Jardim, MS, Brasil.

manejo, preservação e recomposição dessas formações, diante da importância ecológica e beleza cênica que o rio da Prata representa para a região sudoeste do Estado de Mato Grosso do Sul.

\section{Agradecimentos}

Os autores agradecem à CAPES pela bolsa de Mestrado concedida à primeira Autora; à Rede de Sementes do Pantanal, pelo auxílio financeiro; à Universidade Estadual de Mato Grosso do Sul (UEMS), Unidade de Ensino de Jardim, por ceder espaço físico para elaboração deste projeto; à Profa. Dra. Ângela Lúcia Bagnatori Sartori e à Profa. MSc. Ubirazilda Rezende, pela ajuda na identificação do material botânico; à Sra. Elizabeth Secomandi, proprietária da Fazenda Nossa Senhora Aparecida, por autorizar a realização deste estudo em sua propriedade, local denominado "Santuário do Prata", Jardim, MS.

\section{Referências bibliográficas}

Barbosa, L.M. 2000b. Considerações Gerais e Modelos de Recuperação de Formações Ciliares. Pp. 289-312. In: R.R. Rodrigues \& H.F. Leitão-Filho. Matas Ciliares: Conservação e Recuperação. São Paulo, EDUSP/Editora da Universidade de São Paulo.

Bertani, D.F.; Rodrigues, R.R.; Batista, J.L.F. \& Shepherd, G.J. 2001. Análise temporal da heterogeneidade florística e estrutural em uma floresta ribeirinha. Revista Brasileira de Botânica 24(1): 11-23. 
Boggiani, P.C. 1999. Geologia da Bodoquena. Pp. 11-23. In: E. Scremin-Dias; V.J. Pott; R.C. Hora \& P.R. Souza. Nos Jardins Submersos da Bodoquena. Campo Grande, Editora da Universidade Federal de Mato Grosso do Sul.

Borém, R.A.T. \& Oliveira-Filho, A.T. 2002. Fitossociologia do estrato arbóreo em uma topossequiência alterada de mata Atlântica, no município de Silva Jardim-RJ, Brasil. Revista Árvore 26(6): 727-742.

Borges, C.A.; Scheurer-Werle, H.J.; Rosa, D.B.; Paiva, D.J.; Moraes E.P. \& Silva, L.B.S.M. 1997. Geomorfologia. In: Plano de Conservação da Bacia do Alto Paraguai PCBAP, Meio Físico. v.II, Tomo I. Brasília, Ministério do Meio Ambiente, Programa Nacional do Meio Ambiente.

Botrel, R.T.; Oliveira-Filho, A.T.; Rodrigues, L.A. \& Curi, N. 2002. Influência do solo e topografia sobre as variações da composição florística e estrutura da comunidade arbóreo-arbustiva de uma floresta estacional semidecidual em Ingaí, MG. Revista Brasileira de Botânica 25(2): 195-213.

Campelo-Júnior, J.H.; Sandanielo, A.; Caneppele, C.R. \& Soriano, B.M.A. 1997. Climatologia. Pp. 309-349. In: Plano de Conservação da Bacia do Alto Paraguai - PCBAP Meio Físico. V.II, Tomo I. Brasília, Ministério do Meio Ambiente, Programa Nacional do Meio Ambiente.

Campos, J.B. \& Souza, M.C. 2002. Arboreous Vegetation of na Alluvial Riparian Forest and Their Soil Relations: Porto Rico Island, Paraná River, Brazil. Brazilian Archives of Biology and Technology 45(2): 137-149.

Carmo, M.R.B. \& Morellato, L.P.C. 2000. Fenologia de árvores e arbustos das matas ciliares da Bacia do Rio Tibagi, Estado do Paraná, Brasil. Pp. 125-141. In: R.R. Rodrigues \& H.F. Leitão-Filho. Matas Ciliares: conservação e recuperação. São Paulo, EDUSP/Editora da Universidade de São Paulo.

Carvalho, D.A.; Oliveira-Filho, A.T.; Vilela, E.A. \& Curi, N. 2000. Florística e estrutura da vegetação arbórea de um fragmento de floresta semidecidual às margens do reservatório da usina hidrelétrica Dona Rita, Itambé do Mato Dentro, MG. Acta Botanica Brasilica 14(1): 37-55.

Corbacho, C.; Sanchez, J.M. \& Costillo, E. 2003. Patterns of structural complexity and human disturbance of riparian vegetation in agricultural landscapes of a Mediterranean area. Agriculture Ecosystems and Environment 95: 495-507.

Correia, J.R.; Haridasan, M.; Reatto, A.; Martins, E.S. \& Walter, B.M.T. 2001. Influência de fatores edáficos na distribuição de espécies arbóreas em matas de galeria na região do cerrado: uma revisão. Pp. 51-76. In: J.F. Ribeiro; C.E.L. Fonseca \& J.C. Souza-Silva. 2001. Cerrado: caracterização e recuperação de Matas de Galeria. Planaltina, EMBRAPA/Cerrados.

Dasmaceno Júnior, G.; Nakajima, J.N. \& Rezende U.M. 2000. A Floristic survey of the Rio Negro, Rio Aquidauana and Rio Miranda watersheds (Headwaters) of the Pantanal, Mato Grosso do Sul, Brasil. Pp. 34-43. In: P.W. Willink; B. Chernoff; L.E. Alonso; J.R. Montambault \& R. Lourival. A biological assessment of the aquatic ecosystems of the Pantanal, Mato Grosso do Sul, Brasil. Washington, RAP Bulletin of Biological Assessment 18, Conservation International.
EMBRAPA. 1999. Sistema brasileiro de classificação de solos. Rio de Janeiro, Empresa Brasileira de Pesquisa Agropecuária, Centro Nacional de Pesquisa de Solos.

Felfili, J.M.; Mendonça, R.C.; Walter, B.M.T.; Silva Júnior, M.C.; Nóbrega, M.G.G.; Fagg, C.W.; Sevilha, A.C. \& Silva, M.A. 2001. Flora Fanerogâmica das Matas de Galeria e Ciliares do Brasil Central. Pp. 195-263. In: J.F. Ribeiro; C.E.L. Fonseca \& J.C. Souza-Silva. Cerrado: caracterização e recuperação de Matas de Galeria. Planaltina, EMBRAPA/Cerrados.

Ivanauskas, N.M.; Rodrigues, R.R. \& Nave, A.G. 1997. Aspectos ecológicos de um trecho de floresta de brejo em Itatinga, SP: florística, fitossociologia e seletividade de espécies. Revista Brasileira de Botânica 20(2): 139-153.

Ivanauskas, N.M.; Rodrigues, R.R. \& Nave, A.G. 1999. Fitossociologia de um trecho de floresta estacional semidecidual em Itatinga, São Paulo, Brasil. Scientia Florestalis 56: 83-99.

Johnson, M.A.; Saraiva, P.M. \& Coelho, D. 1999. The role of gallery forests in the distribution of Cerrado mammals. Revista Brasileira de Biologia 59(3): 421-427.

Kageyama, P. \& Gandara, F.B. 1998. Conseqüências genéticas da fragmentação sobre populações de espécies arbóreas. Série Técnica IPEF 12(32): 65-70.

Kageyama, P. \& Gandara, F.B. 2000. Recuperação de Áreas Ciliares. Pp. 249-269. In: R.R. Rodrigues \& H.F. LeitãoFilho. 2000. Matas Ciliares: conservação e recuperação. São Paulo, EDUSP/Editora da Universidade de São Paulo.

Leitão-Filho, H.F. 1982. Aspectos taxonômicos das florestas do Estado de São Paulo. Silvicultura em São Paulo 1: 197-206.

Lima, M.G. \& Gascon, C. 1999. The conservation value of linear forest remnants in central Amazonia. Biological Conservation 91: 241-247.

Lima, W.P. \& Zakia, M.J.B. 2000. Hidrologia de Matas Ciliares. Pp. 33-44. In: R.R. Rodrigues \& H.F. Leitão-Filho. 2000. Matas Ciliares: conservação e recuperação. São Paulo, EDUSP/Editora da Universidade de São Paulo.

Lobo, P.C. \& Joly, C.A. 2000. Aspectos ecofisiológicos da vegetação de mata ciliar do sudeste do Brasil. Pp. 143-157. In: R.R. Rodrigues \& H.F. Leitão-Filho. Matas Ciliares: conservação e recuperação. São Paulo, EDUSP/Editora da Universidade de São Paulo.

Lorenzi, H. 1998. Árvores Brasileiras - manual de identificação e cultivo de plantas arbóreas nativas do Brasil. 2 ${ }^{\mathrm{a}}$ ed. v.2. Nova Odessa, Instituto Plantarum.

Lorenzi, H. 2000. Árvores Brasileiras - manual de identificação e cultivo de plantas arbóreas nativas do Brasil. $3^{\mathrm{a}}$ ed. v.1. Nova Odessa, Instituto Plantarum.

Lüttge, U. 1997. Physiological Ecology of Tropical Plants. Berlin, Springer-Verlag, Heidelberg. Germany.

Magurran, A.E. 1988. Ecological Diversity and its Measurement. Princeton University Press.

Montag, L.F.A.; Smith, W.S.; Barrella, W. \& Petrere Jr., M. 1997. As influências e as relações das matas ciliares nas comunidades de peixes do Estado de São Paulo. Revista Brasileira de Ecologia 1: 76-80. 
Morelli, S.L. 2000. Legislação Ambiental do Estado de Mato Grosso do Sul. Campo Grande, Editora da Universidade Federal de Mato Grosso do Sul.

Nathan, R. \& Landau, M.C.H. 2000. Spatial patterns of seed dispersal, their determinants and consequences for recruitment. Tree 15(7): 278-285.

Nebel, G.; Kvist, L.P.; Vanclay, J.K.; Christensen, H.; Freitas, L. \& Ruíz, J. 2001. Structure and floristic composition of flood plain forests in the Peruvian Amazon I. Overstorey. Forest Ecology and Management 150: 27-57.

Nunes, Y.R.F.; Mendonça, A.V.R.; Botezelli, L.; Machado, E.L.M. \& Oliveira-Filho, A.T. 2003. Variações da fisionomia, diversidade e composição de guildas da comunidade arbórea em um fragmento de floresta semidecidual em Lavras, MG. Acta Botanica Brasilica 17(2): 213-229.

Oliveira-Filho, A.T. \& Ratter, J. 2000. Padrões florísticos das Matas Ciliares da Região do Cerrado e a evolução das paisagens do Brasil Central durante o Quaternário Tardio. Pp. 73-89. In: R.R. Rodrigues \& H.F. LeitãoFilho. Matas Ciliares: conservação e recuperação. São Paulo, EDUSP/Editora da Universidade de São Paulo.

Oliveira-Filho, A.T.; Almeida, R.J.; Mello, J.M. \& Gavilenes, M.L. 1994. Estrutura fitossociológica e variáveis ambientais em um trecho da mata ciliar do córrego dos Vilas-Boas, Reserva Ecológica do Poço Bonito, Lavras (MG). Revista Brasileira de Botânica 17: 67-85.

Pagano, S.N.; Leitão-Filho, H.F. \& Cavassan, O. 1995. Variação temporal da composição florística e estrutura fitossociológica de uma floresta mesófila semidecídua, Rio Claro, Estado de São Paulo. Revista Brasileira de Biologia 55(2): 241-258.

Paine, L.K. \& Ribic, C.A. 2002. Comparison of riparian plant communities under four land management systems in southwestern Wisconsin. Agriculture Ecosystems Environment 92: 93-105.

Pott, A. \& Pott, V.J. 1994. Plantas do Pantanal. Corumbá, EMBRAPA.

Pott, A. \& Pott, V.J. 2003. Espécies de Fragmentos Florestais em Mato Grosso do Sul. Pp. 26-52. In: R.B. Costa. Fragmentação florestal e alternativas de desenvolvimento rural na região Centro-Oeste. Campo Grande, Universidade Católica Dom Bosco.

Ribeiro, J.F. \& Walter, B.M.T. 1998. Fitofisionomias do Bioma Cerrado. Pp. 89-166. In: S.M. Sano \& S.P. Almeida. Cerrado: ambiente e flora. Planaltina, EMBRAPA/ Cerrados.
Ribeiro, J.F. \& Walter, B.M.T. 2001. As Matas de Galeria no contexto do bioma Cerrado. Pp. 29-47. In: J.F. Ribeiro; C.E.L. Fonseca \& J.C. Souza-Silva. Cerrado: caracterização e recuperação de Matas de Galeria. Planaltina, EMBRAPA/Cerrados.

Rodrigues, R.R. \& Nave, A.G. 2000. Heterogeneidade florística das matas ciliares. Pp. 45-71. In: R.R. Rodrigues \& H.F. Leitão-Filho. Matas Ciliares: conservação e recuperação. São Paulo, EDUSP/Editora da Universidade de São Paulo.

Romagnolo, M.B. \& Souza, M.C. 2000. Análise florística e estrutural de florestas ripárias do Alto Rio Paraná, Taquaruçu, MS. Acta Botanica Brasilica 14(2): 163-174.

Romagnolo, M.B. \& Souza, M.C. 2004. Os gêneros Calycorectes O. Berg, Hexachlamys O. Berg, Myrcianthes O. Berg, Myrciaria O. Berg e Plinia L. (Myrtaceae) na planície alagável do alto rio Paraná, Brasil. Acta Botanica Brasilica 18(3): 613-627.

Salis, S.M.; Tamashiro, J.Y. \& Joly, C.A. 1994. Florística e fitossociologia do estrato arbóreo de um remanescente de mata ciliar do rio Jacaré-Pepira, Brotas, SP. Revista Brasileira de Botânica 17(2): 93-103.

Sampaio, A.B.; Walter, B.M.T. \& Felfili, J.M. 2000. Diversidade e distribuição de espécies arbóreas em duas matas de galeria na micro-bacia do Riacho Fundo, Distrito Federal. Acta Botanica Brasilica 14(2): 197-214.

Sanchez, M.; Pedroni, F.; Leitão-Filho, H.F. \& Cesar, O. 1999. Composição florística de um trecho de floresta ripária na Mata Atlântica em Picinguaba, Ubatuba, SP. Revista Brasileira de Botância 22(1): 31-42.

Shepherd, G.J. 1996. FITOPAC versão 2.0. Campinas, Universidade Estadual de Campinas.

Silva Júnior, M.C.; Felfili, J.M.; Nogueira, P.E. \& Rezende, A.V. 1998. Análise Florística das Matas de Galeria no Distrito Federal. Pp. 53-84. In: J.F. Ribeiro. Cerrado: Matas de Galeria. Planaltina, EMBRAPA/Cerrados.

Silva Júnior, M.C. 2001a. Comparação entre matas de galeria no Distrito Federal e a efetividade do código florestal na proteção de sua diversidade arbórea. Acta Botanica Brasilica 15(1): 139-146.

Silva Júnior, M.C.; Felfili, J.M.; Walter, B.M.T.; Nogueira, P.E.; Rezende, A.V.; Morais, R.O. \& Nóbrega, M.G.G. 2001b. Análise da flora arbórea de Matas de Galeria no Distrito Federal: 21 levantamentos. Pp. 143-191. In: J.F. Ribeiro; C.E.L. Fonseca \& J.C. Souza-Silva. Cerrado: caracterização e recuperação de Matas de Galeria. Planaltina, EMBRAPA/Cerrados.

Wang, B.C. \& Smith, T.B. 2002. Closing the seed dispersal loop. Trends in Ecology \& Evolution 17: 379-385.

Whittaker, R.H. 1972. Evolution and measurement of species diversity. Taxon 21: 213-251. 